\title{
Effects of salt substitute on pulse wave analysis among individuals at high cardiovascular risk in rural China: a randomized controlled trial
}

\author{
Jihong $\mathrm{Hu}^{1,2}$, Xiongjing Jiang ${ }^{1}$, Nicole $\mathrm{Li}^{3}$, Xuequn $\mathrm{Yu}^{4}$, Vlado Perkovic ${ }^{3}$, Bailing Chen ${ }^{1}$, Liancheng Zhao ${ }^{1}$, \\ Bruce $\mathrm{Neal}^{3}$ and Yangfeng $\mathrm{Wu}^{1,4,5}$
}

Reduced-sodium, increased-potassium salt substitutes lower blood pressure but may also have direct effects on vascular structure and arterial function. This study aimed to test the effects of long-term salt substitution on indices of these outcomes. The China Salt Substitute Study was a randomized, controlled trial designed to establish the effects of salt substitute $(65 \%$ sodium chloride, $25 \%$ potassium chloride, $10 \%$ magnesium sulfate) compared with regular salt ( $100 \%$ sodium chloride) on blood pressure among 600 high-risk individuals living in six rural areas in northern China over a 12-month intervention period. Data on central aortic blood pressure, aortic pressure augmentation (AUG), augmentation index (AIx), the differences of the peak of first and baseline waves $\left(P_{1}-P_{0}\right)$ and pulse wave reflection time (RT) were collected at randomization and at the completion of follow-up in 187 participants using the Sphygmocor pulse wave analysis system. Mean baseline blood pressure was $150.1 / 91.4 \mathrm{~mm} \mathrm{Hg}$, mean age was 58.4 years, $41 \%$ were male and three quarters had a history of vascular disease. After 12 months of intervention, there were significant net reductions in peripheral $(7.4 \mathrm{~mm} \mathrm{Hg}, P=0.009)$ and central $(6.9 \mathrm{~mm} \mathrm{Hg}$, $P=0.011)$ systolic blood pressure levels and central pulse pressure $(4.5 \mathrm{~mm} \mathrm{Hg}, P=0.012)$ and correspondingly there was a significant net reduction in $P_{1}-P_{0}(3.0 \mathrm{~mm} \mathrm{Hg}, P=0.007)$, borderline significant net reduction in AUG $(1.5 \mathrm{~mm} \mathrm{Hg}, P=0.074)$ and significant net increase in RT $(2.59 \mathrm{~ms}, P=0.001)$. There were no detectable reductions in peripheral $(2.8 \mathrm{~mm} \mathrm{Hg}, P=0.14)$ or central $(2.4 \mathrm{~mm} \mathrm{Hg}, P=0.13)$ diastolic blood pressure levels or Alx $(0.06 \%, P=0.96)$. In conclusion, over the 12-month study period the salt substitute significantly reduced not only peripheral and central systolic blood pressure but also reduced arterial stiffness.

Hypertension Research (2009) 32, 282-288; doi:10.1038/hr.2009.7; published online 27 February 2009

Keywords: central aortic blood pressure; pulse wave analysis; randomized trial; salt substitute; wave reflection

\section{INTRODUCTION}

Central aortic blood pressure levels are determinants of cardiac loading and perfusion. They impact significantly on cardiovascular function and increased pulse pressure reflects stiffening of conduit vessels. ${ }^{1}$ Elevated pulse pressure is associated with an increased risk for coronary artery disease, ${ }^{2}$ stroke $^{3}$ and the development and progression of heart failure. ${ }^{4}$ In addition to the pressure levels, the arterial pressure waveform conveys information regarding systemic vascular stiffness. $^{5}$ Arterial stiffness increases with age, causes increased left ventricular after load and is an independent predictor of cardiovascular morbidity and mortality. ${ }^{6,7}$ The aortic augmentation index (AIx) is widely used as an indicator of arterial stiffness and itself is a strong predictor of change in left ventricular mass index ${ }^{8}$ and mortality. ${ }^{9}$ The European Society of Hypertension recommends that the evaluation of new blood pressure-lowering treatments should include assessment of effects on novel hemodynamic factors such as arterial stiffness. ${ }^{10}$

Excess salt consumption is widely considered to be a leading cause of both short and long-term increases in blood pressure ${ }^{11,12}$ and effects may be mediated through both volume expansion and changes in arterial structure and function. Salt substitutes with reduced sodium and increased potassium substantially lower blood pressure. ${ }^{13-15}$ There are some limited data to suggest that long-term consumption of a diet low in salt may reduce arterial stiffness independent of blood pressure reduction ${ }^{16}$ although data from high-risk individuals with existing vascular disease are few. There are also data showing positive correlations of sodium intake ${ }^{17}$ and negative correlations of potassium intake ${ }^{18}$ with arterial stiffness

${ }^{1}$ Cardiovascular Institute and Fuwai Hospital, Chinese Academy of Medical Sciences and Peking Union Medical College, Beijing, China; ${ }^{2}$ Department of Public Health, Ningxia Medical College, Ningxia, China; ${ }^{3}$ The George Institute for International Health, Sydney, Australia; ${ }^{4}$ The George Institute China, Beijing, China and ${ }^{5}$ Department of Epidemiology and Biostatistics, Peking University School of Public Health, Beijing, China

Correspondence: Dr Y Wu, Peking University School of Public Health, No. 38 Xueyuanlu, Haidian District, Beijing 100083, PR China.

E-mail: wuyf@bjmu.edu.cn

Received 8 July 2008Received 22 December 2008; accepted 16 January 2009; published online 27 February 2009 
providing a strong rationale for beneficial effects of salt substitution beyond blood pressure lowering although once again data about effects in high-risk individuals are limited. In this study done among a subset of participants in the China Salt Substitute Study (CSSS) ${ }^{19}$ most of who had evidence of existing vascular disease, we sought to determine the long-term effects of salt substitute on arterial stiffness and to test the hypothesis that salt substitution has medium term effects beyond just volume depletion in this patient group.

\section{METHODS}

The design and results of the CSSS have been published previously. ${ }^{19}$ In brief, the study was a double-blind, randomized, controlled trial which clearly demonstrated the blood pressure lowering effects of a reduced-sodium, highpotassium salt substitute among 607 individuals at elevated risk of cardiovascular disease living in rural China. The study was done between May 2004 and August 2005 at 39 sites distributed among six regional coordinating centers in Northern China (Heilongjiang, Tianjin, Liaoning, Shanxi and two centers in Beijing). All participants in the main trial and the 187 in this substudy (conducted at the two centers in rural/suburban Beijing) provided informed consent. The main trial received ethic approvals from the University of Sydney, Australia and the Fu Wai Hospital, Beijing China and this substudy approval from Fu Wai Hospital. The trial was registered with http://clinicaltrials.gov/ with the Identifier: NCT00145756.

\section{Participants}

Individuals were eligible for the study if they had a high risk of future vascular disease based on a doctor's diagnosis of any of the following: (1) coronary, cerebral or peripheral vascular disease, (2) diabetes and aged 55 years or older or (3) a systolic blood pressure $\geqslant 160 \mathrm{~mm} \mathrm{Hg}$. In addition all participants were required to have an estimated daily sodium intake of $260 \mathrm{mmol} \mathrm{day}^{-1}$ or more and an expectation that at least half of the dietary salt could be replaced with the study salt-substitute/salt. This was done in an effort to recruit a group with an above average salt consumption and thereby maximize the power of the study to detect effects of the salt substitute on the various outcome measures. Finally participants were required to have no established clear indication for, or contra-indication to, the use of the study salt substitute, such as use of a potassium-sparing medication or significant renal impairment. A blood test was performed at registration and randomization to check serum creatinine and potassium levels before and after the active 1 month run-in period, during which all potential participants used the salt substitute. Any individual with a blood test result considered by the responsible physician to be possibly abnormal was excluded.

\section{Randomization}

Randomization was undertaken after the one-month run-in period using a central computerized randomization service accessed by the center physicians through the study website, with a back up phone and fax service. The service was maintained by the Clinical Trials Research Unit at the University of Auckland, New Zealand. Randomization was stratified within each center by baseline systolic blood pressure $(<140 \mathrm{~mm} \mathrm{Hg}, 140-160 \mathrm{~mm} \mathrm{Hg}$, $>160 \mathrm{~mm} \mathrm{Hg}$ ) and current use of antihypertensive therapy. The randomization service provided a unique number for each individual corresponding to a treatment pack held at the center. Treatment allocation was blinded to study investigators, participants and center physicians until the study database was locked.

\section{Study treatment}

Salt substitute was given to all participants for the run-in period and then either salt substitute or normal salt to those participants who were randomized (Figure 1). The salt substitute was $65 \%$ sodium chloride, $25 \%$ potassium chloride and $10 \%$ magnesium sulfate. This formulation is commercially available in China and had been tested previously. ${ }^{13}$ The normal salt was $100 \%$ sodium chloride. Randomized treatment was delivered in $1 \mathrm{~kg}$ bags identical except for a 3-digit code corresponding to the randomization number, with up to $3 \mathrm{~kg}$ a month salt substitute/salt available to each randomized participant to cover all cooking, pickling and other uses within the household. Participants were instructed to use the study salt for all food preparation throughout the study duration.

\section{Data collection}

Potentially eligible individuals attended a registration visit with the physician at the center where informed consents were obtained, a baseline questionnaire was completed, a brief physical examination was performed and a blood sample was collected. Individuals consenting to participate in the trial were entered into the active run-in period and instructed to return for a final eligibility check 4 weeks later. Eligible participants who attended the next scheduled visit were randomized. Peripheral blood pressure measurements were made at registration, randomization, 1, 2, 3, 6, 9 and 12 months post randomization visits, and then centrally entered into a password-protected study website in Beijing. Additional data on central aortic blood pressure, aortic pressure augmentation (AUG) and AIx were collected at the randomization visit and the 12-month follow up visit.

\section{Outcomes}

The primary outcomes of the pulse wave analysis were central aortic blood pressure including central systolic blood pressure (CSP) and central diastolic blood pressure (CDP) and central pulse pressure (CPP), P1, reflection time (RT), AUG and AIx. The analysis was of central waveforms derived from radial measurements. CPP and $\mathrm{P}_{1}$ were respectively computed by the following formulas: $\mathrm{CPP}=\mathrm{CSP}-\mathrm{CDP}$ and $\mathrm{P}_{1}=\mathrm{CSP}-\mathrm{AUG}$. The secondary outcomes included peripheral systolic blood pressure (PSP) and peripheral diastolic blood pressure (PDP) (Figure 2).

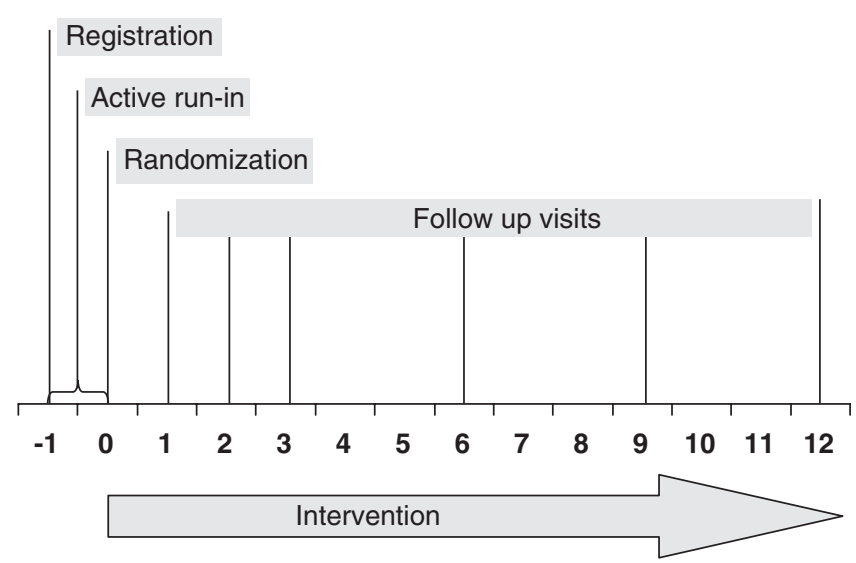

Figure 1 The study design.

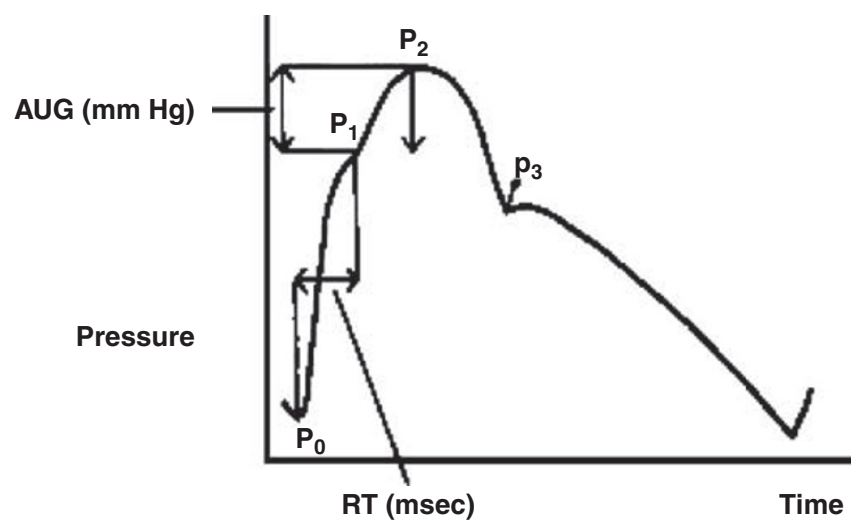

Figure 2 The illustration of the pulse wave and the augmentation index. AUG (aortic pressure augmentation) $=P_{2}-P_{1} . P_{2}$ is the peak of secondary waves, in other words, central aortic systolic blood pressure. $P_{1}$ is the peak of first wave. $P_{0}$ is central diastolic blood pressure. RT means 'Reflection Time'. 
Peripheral blood pressure was recorded using an OMRON HEM-770A automatic sphygmomanometer and was measured in the right arm with participants seated at rest for at least $5 \mathrm{~min}$ beforehand. The mean of two measurements made at least $2 \mathrm{~min}$ apart was used for analysis. Validity of the Omron HEM-770A electronic device against a calibrated mercury sphygmomanometer was done according to British Hypertension Society guidelines among 85 volunteers aged 15 to 80 prior to commencement of the study with the Omron meeting grade A BHS-criteria.

Central aortic blood pressure, AUG and AIx were estimated through noninvasive pulse waveform analysis using the Sphygmocor device (AtCor Medical Ltd., Australia). ${ }^{7}$ After an overnight fast, participants were seen at the clinic between 0800 and 1000 hours and rested in a sitting position for at least $5 \mathrm{~min}$ before the pulse waveform analysis measurements were undertaken. Peripheral pressure waveforms were recorded from the radial artery at the wrist, using applanation tonometry with a high-fidelity micromanometer. The parameters indicating wave reflection were AUG and AIx. AUG was defined as the difference between the peaks of the secondary and primary waves $\left(A U G=P_{2}-P_{1}\right)$. AIx was defined as the ratio of AUG to the differences of the peak of secondary and baseline waves $\left(\mathrm{AI}=\mathrm{AUG} /\left(\mathrm{P}_{2}-\mathrm{P}_{0}\right)\right)$. After 20 sequential waveforms had been acquired, a validated, mathematic transfer function was used to generate the corresponding central aortic pressure waveform and central hemodynamic parameters including AIx, CSP, CDP and CPP. ${ }^{20}$ This method was previously validated by invasive measurements. ${ }^{21}$ Only high quality recordings, defined as an in-device quality index $\geqslant 80 \%$ and acceptable curves on visual inspection by the investigator, were considered valid and data from five patients were excluded as they did not meet these criteria. Wave reflection was recorded two times at each clinical visit and the mean of two valid measurements was used for analysis. All pulse wave measurements were done by one person, trained by an expert. A test-retest study done in 20 individuals to determine the reproducibility of augmentation index measurements showed a difference of $0.3 \%$ (s.d. $0.8 \%, P=0.2$ ) indicating that pulse wave measurement was highly reproducible.

\section{Statistical method}

We used data collected at the randomization and 12-month visits to evaluate the effects of the intervention on the primary outcomes but used data from all visits to plot the figure showing changes in PSP and PDP during the trial. Intention to treat analysis was used and all analysis was performed using the SPSS statistical package, version 11.5 (SPSS Inc., Chicago, IL, USA). Student's paired tests were used to test the changes from randomization to follow-up within each of the two randomized groups and student's unpaired tests to compare the differences between the two groups. For continuous variables that were not normally distributed the analysis was done using either the MannWhitney $U$-test or the Wilcoxon test. The Analysis of Variance method was used to compare the changes between groups in blood pressure from randomization to 12 months with adjustment for the baseline value. $\chi^{2}$ tests were used to compare the proportions of categorical variables between groups. A probability of $P<0.05$ was considered statistically significant.

\section{RESULTS}

In the two participating centers in Beijing there were a total of 200 participants registered and entered into the run-in phase and there were 192 (96\%) individuals randomized (Figure 3). The reasons why the eight participants were excluded during run-in were stroke $(n=1)$, reluctance to continue $(n=3)$ or other unknown reasons $(n=4)$. All

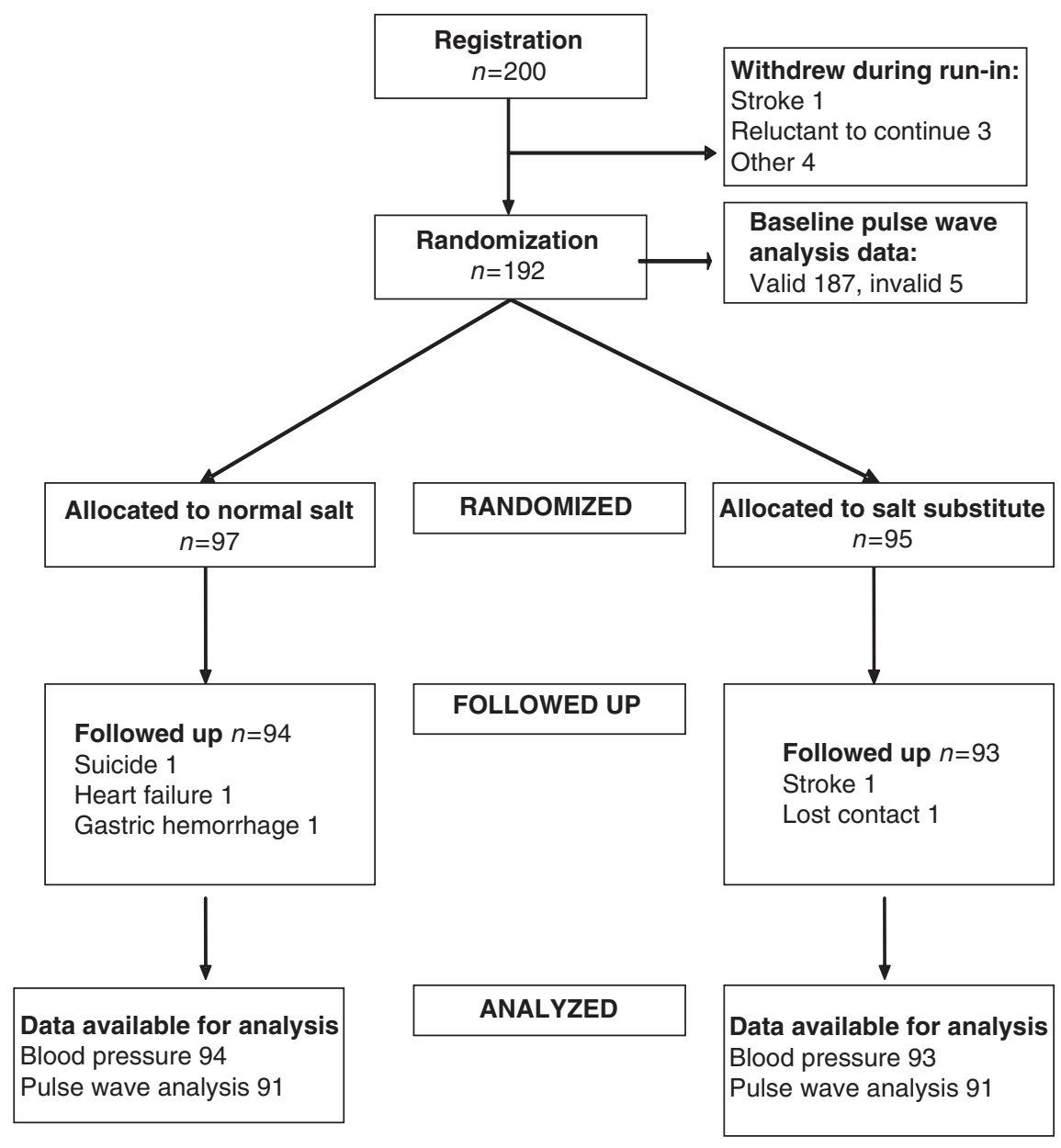

Figure 3 Flow chart. 
192 participants had baseline measurements of the primary outcome but five assessments (regular salt group=3, salt substitute group=2) were subsequently found to be of inadequate quality leaving 187 valid baseline pulse wave records. Three people in the control group were not available for 12-month follow-up measurements (suicide $\times 1$, heart failure $\times 1$, gastric hemorrhage $\times 1$ ) as were two people in the salt substitute group $($ stroke $\times 1$, relocation $\times 1)$. As such 182 of the 192 randomized participants had complete and valid wave reflection records at randomization and follow-up (94\%).

The mean age of randomized participants was $58.4 \pm 9.6$ years, $41 \%$ were male and $77 \%$ had a baseline history of vascular disease. All participants reported that they used study salt substitute/salt for 'all' or 'nearly all' of their day to day food preparation with no difference between randomized groups $(P=0.88)$. Key baseline characteristics including SBP and DBP were balanced between the randomized groups (all $P>0.05$ ) (Table 1) and the mean baseline BP was 0.150.1/91.4 mm Hg (s.d. 23.2/13.0 mm Hg).

The mean peripheral blood pressure difference between randomized groups at the end of the trial was $7.4 \mathrm{~mm} \mathrm{Hg}$ (95\% confidence interval $1.9-13.0 \mathrm{~mm} \mathrm{Hg} ; P=0.009)$ systolic and $2.3 \mathrm{~mm} \mathrm{Hg}(0.7-5.3 \mathrm{~mm} \mathrm{Hg}$; $P=0.139)$ diastolic. The changes of mean peripheral blood pressure

Table 1 Characteristics of study participants at baseline and randomization by randomized group ${ }^{\mathrm{a}}$

\begin{tabular}{|c|c|c|}
\hline & Salt substitute & Normal salt \\
\hline Male & $43(46 \%)$ & $33(35 \%)$ \\
\hline Mean age (years) (s.d.) & $59(10.0)$ & $59(9.1)$ \\
\hline \multicolumn{3}{|l|}{ Inclusion criteria } \\
\hline History of vascular disease & $72(77 \%)$ & $68(72 \%)$ \\
\hline $\mathrm{SBP} \geqslant 160 \mathrm{~mm} \mathrm{Hg}$ & $32(34 \%)$ & $37(39 \%)$ \\
\hline Treated diabetes and age $>55$ & $20(22 \%)$ & $27(29 \%)$ \\
\hline \multicolumn{3}{|l|}{ Characteristics at baseline } \\
\hline $\begin{array}{l}\text { Mean blood pressure (SBP/DBP), } \\
\mathrm{mm} \mathrm{Hg} \mathrm{(s.d.)}\end{array}$ & $\begin{array}{l}149.4 / 91.0 \\
(22.3 / 12.8)\end{array}$ & $\begin{array}{l}150.9 / 91.8 \\
(24.2 / 13.3)\end{array}$ \\
\hline \multicolumn{3}{|l|}{ Concomitant drug treatments } \\
\hline Antihypertensive agents & $69(74 \%)$ & $66(70 \%)$ \\
\hline Diuretic & $4(4 \%)$ & $3(3 \%)$ \\
\hline Calcium antagonist & $31(33 \%)$ & $31(33 \%)$ \\
\hline ACE inhibitor or ARB & $8(9 \%)$ & $13(14 \%)$ \\
\hline$\beta$-blocker & $7(8 \%)$ & $2(2 \%)$ \\
\hline Other & $39(42 \%)$ & $44(47 \%)$ \\
\hline Nitrates & $3(3 \%)$ & $2(2 \%)$ \\
\hline Mean body mass index, $\mathrm{kg} / \mathrm{m}^{2}$ (s.d.) & $27(3.9)$ & $26(3.8)$ \\
\hline \multicolumn{3}{|l|}{ Characteristics at randomization } \\
\hline $\begin{array}{l}\text { Mean blood pressure (SBP/DBP), } \\
\mathrm{mm} \mathrm{Hg} \text { (s.d.) }\end{array}$ & $\begin{array}{l}139.2 / 86.5 \\
(21.6 / 12.7)\end{array}$ & $\begin{array}{l}139.9 / 87.1 \\
(22.2 / 12.3)\end{array}$ \\
\hline Mean aortic blood pressure, & $128.6 / 88.0$ & $129.8 / 88.7$ \\
\hline $\mathrm{mm} \mathrm{Hg}$ (s.d.) & $(20.1 / 13.0)$ & $(20.3 / 12.6)$ \\
\hline Mean heart rates, beats/minute (s.d.) & $75.2(10)$ & $75.6(12)$ \\
\hline CPP, mm Hg (s.d.) & $40.4(12.6)$ & $41.1(13.3)$ \\
\hline P1, mm Hg (s.d.) & $118.1(17.1)$ & $118.7(17.3)$ \\
\hline Mean AUG, mm Hg (s.d.) & $10.4(6.1)$ & $11.1(6.3)$ \\
\hline Mean Alx (s.d.) & $24.0(9.3)$ & $25.1(9.4)$ \\
\hline RT, msec (s.d.) & $106.7(14.1)$ & 109.4 (13.8) \\
\hline
\end{tabular}

Abbreviations: ACE, angiotensin converting enzyme; Alx, aortic pressure augmentation index ARB, angiotensin receptor blocker; AUG, aortic pressure augmentation; CPP, central pulse pressure; DBP, diastolic blood pressure; RT, reflection time; SBP, systolic blood pressure. anumber (percent) unless otherwise indicated. during 12 months of intervention were illustrated in Figure 4. Table 2 summarizes mean values (s.d.) of hemodynamic parameters at randomization and 12-month visit and the changes in each group. Comparison of the changes between groups was also made. To adjust for the effect of baseline levels on the changes, the net changes of each variable from randomization to the 12-month visit between salt and salt substitute groups were calculated with multiple variable analysis of variance (Figure 5). The results showed that there were significant net reductions in central systolic blood pressure levels (mean 6.9, 95\% confidence interval $1.6-12.3 ; P=0.011$ ), mean central pulse pressure $(4.5,1.0-8.0 ; P=0.012)$, mean $\mathrm{P}_{1}-\mathrm{P}_{0}(3.0,0.8-5.2 ; P=0.007)$, and $\mathrm{a}$ borderline net reduction in AUG $(1.5 \mathrm{~mm} \mathrm{Hg},-0.1-3.2 ; P=0.074)$ but there was a significant increase in RT $(2.59 \mathrm{~ms}, 0.76-4.42$; $P=0.001)$ in salt substitute group. There were no detectable reductions in central diastolic blood pressure levels $(2.4 \mathrm{~mm} \mathrm{Hg},-0.7-5.5$; $P=0.13)$ or AIx $(0.06 \%,-2.2-2.3 ; P=0.96)$.

There were no significant differences in the proportion of participants using drugs for blood pressure lowering at baseline or follow-up (data not shown). Repeating all analyses in those patients who either never used blood pressure lowering drugs or used such drugs constantly throughout the study did not produce different results (data not shown).

\section{DISCUSSION}

This study performed using a subset of participants in the main China Salt Substitute Study ${ }^{19}$ adds substantially to our understanding of the mechanism by which a reduced sodium, added potassium salt substitute affects arterial hemodynamics in patients with established vascular disease. Specifically, the assessments completed here show that the short to medium term use of the salt substitute are not only reducing blood pressure, both peripheral and central, but also arterial stiffness after 12 months of intervention. To our knowledge, this is the

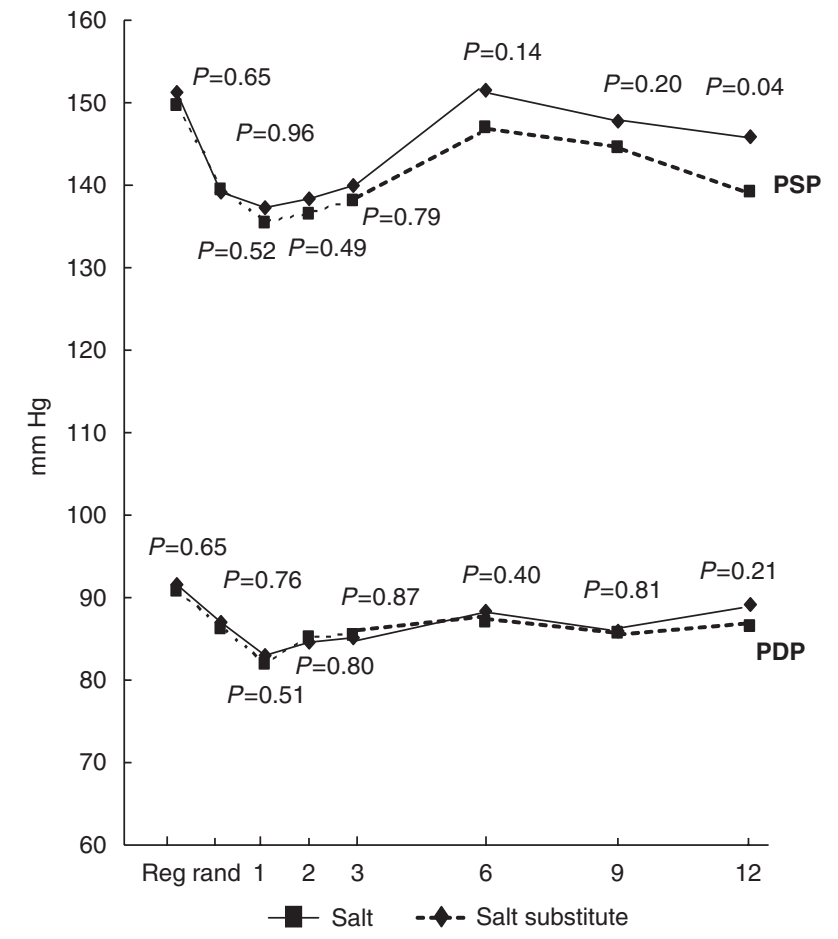

Figure 4 Mean levels of peripheral SBP and DBP at each visit by treatment group $(\mathrm{mm} \mathrm{Hg})$. 
Table 2 Mean values (s.d.) of hemodynamic parameters at randomization and 12-month visit

\begin{tabular}{|c|c|c|c|c|c|c|c|c|c|}
\hline & \multicolumn{4}{|c|}{ Normal salt group $(\mathrm{n}=94)$} & \multicolumn{5}{|c|}{ Salt substitute group $(\mathrm{n}=93)$} \\
\hline & At randomization & At 12 month & Change & $P^{*}$ & At randomization & At 12 month & Change & $P^{*}$ & P\# \\
\hline Peripheral DBP $(\mathrm{mm} \mathrm{Hg})$ & $87.1(12.3)$ & $89.2(13.1)$ & $2.1 \pm 11.4$ & 0.085 & $86.5 \pm 12.7$ & $86.6 \pm 13.8$ & $0.1 \pm 10.6$ & 0.984 & 0.227 \\
\hline \multicolumn{10}{|l|}{ Heart rate } \\
\hline (beats/minute) & $75.6 \pm 12.0$ & $73.1 \pm 10.8$ & $-2.5 \pm 10.3$ & 0.023 & $75.2 \pm 10.3$ & $73.2 \pm 10.5$ & $-2.0 \pm 8.5$ & 0.028 & 0.664 \\
\hline $\mathrm{CPP}(\mathrm{mm} \mathrm{Hg})$ & $41.1 \pm 13.3$ & $46.3 \pm 14.8$ & $5.2 \pm 15.1$ & 0.002 & $40.4 \pm 12.6$ & $41.4 \pm 13.3$ & $1.0 \pm 10.6$ & 0.432 & 0.031 \\
\hline RT (msec) & $109.4 \pm 13.8$ & $110.6 \pm 13.7$ & $1.24 \pm 16.4$ & 0.474 & $106.7 \pm 14.1$ & $110.6 \pm 13.3$ & $3.93 \pm 13.8$ & 0.986 & 0.235 \\
\hline $\mathrm{P} 1(\mathrm{~mm} \mathrm{Hg})$ & $118.7 \pm 17.3$ & $123.7 \pm 18.6$ & $5.0 \pm 17.3$ & 0.007 & $118.1 \pm 17.2$ & $117.8 \pm 18.2$ & $-0.3 \pm 14.5$ & 0.754 & 0.028 \\
\hline Augment pressure $(\mathrm{mm} \mathrm{Hg})$ & $11.1 \pm 6.3$ & $13.5 \pm 6.9$ & $2.4 \pm 7.0$ & 0.001 & $10.4 \pm 6.1$ & $11.7 \pm 6.1$ & $1.3 \pm 5.7$ & 0.036 & 0.228 \\
\hline $\mathrm{P}_{1}-\mathrm{P}_{0}(\mathrm{~mm} \mathrm{Hg})$ & $30.0 \pm 8.5$ & $32.7 \pm 9.3$ & $2.7 \pm 9.5$ & 0.008 & $30.0 \pm 8.2$ & $29.7 \pm 8.7$ & $0.3 \pm 6.5$ & 0.121 & 0.013 \\
\hline Alx $(\%)$ & $25.1 \pm 9.4$ & $27.4 \pm 9.1$ & $2.3 \pm 9.7$ & 0.027 & $24.0 \pm 9.3$ & $26.9 \pm 8.4$ & $2.9 \pm 8.9$ & 0.003 & 0.657 \\
\hline
\end{tabular}

Abbreviations: $\mathrm{Alx}=$ aortic pressure augmentation index; $\mathrm{CPP}=$ central pulse pressure; $\mathrm{DBP}=$ diastolic blood pressure; $\mathrm{HR}=$ heart rate; $\mathrm{RT}=$ reflection time; $\mathrm{SBP}=$ systolic blood pressure. $P_{1}$ was the peak of first waves, $P_{1}=P_{2}-A U G$.

$P_{0}$ was central diastolic blood pressure.

$P^{*}$ for changes before and after intervention comparisons.

$\mathrm{P \#}$ for comparisons of the changes between intervention groups.

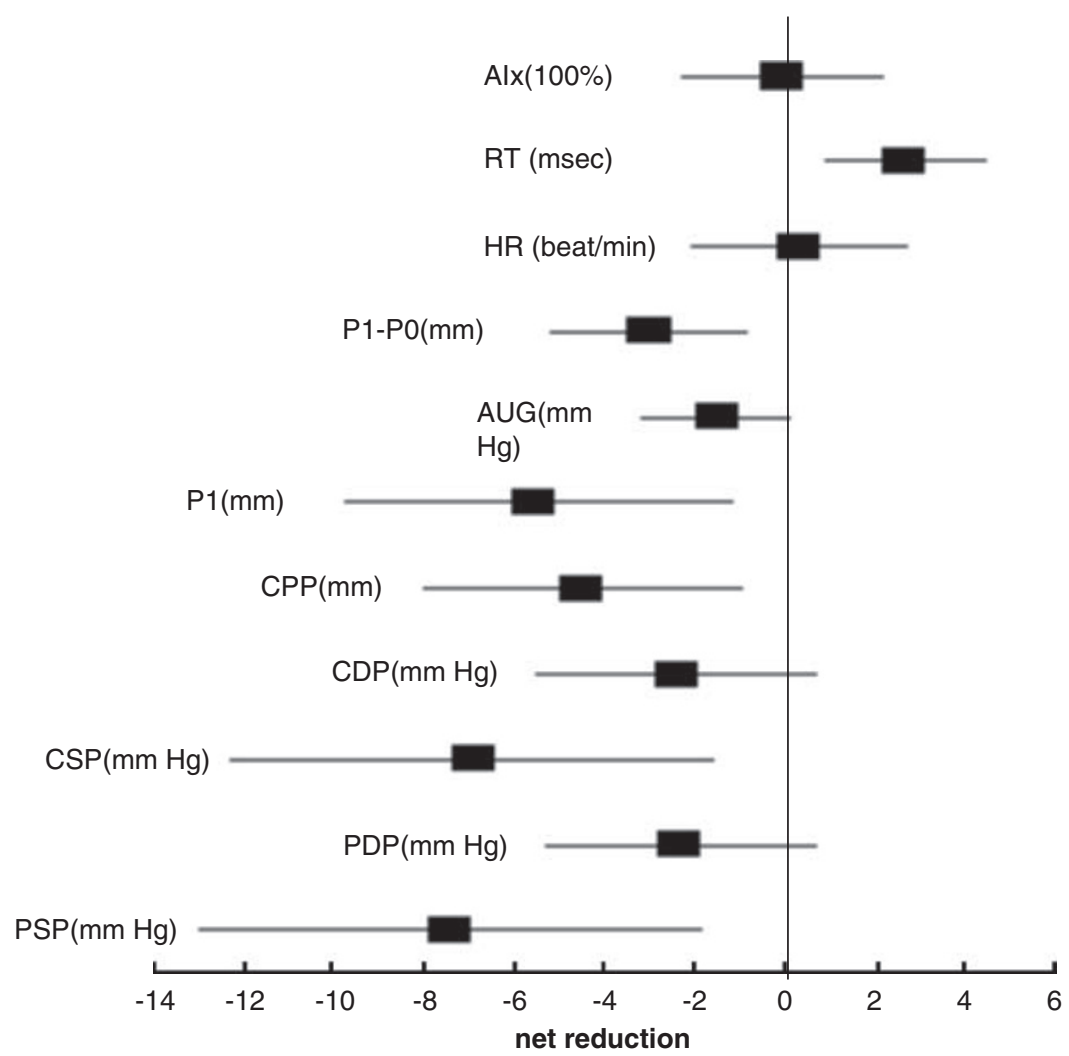

Figure 5 Net reductions of hemodynamic parameters between groups after adjusting for baseline values.

first report that found such effect of a low sodium and high-potassium salt substitute on arterial stiffness.

Prior small scale intervention studies of effects of dietary sodium restriction shows that salt intake is associated with arterial stiffness ${ }^{16,22}$ and the study done by Stewart et al. ${ }^{23}$ shows that acute reduction of blood pressure by nitroglycerin normalizes large artery stiffness in normortensives but does not normalize that in essential hypertension, suggesting that the latter group requires long-term intervention to change arterial stiffness. Studies of pharmacological blood pressure lowering interventions have also shown the similar effects. ${ }^{24-26}$ Diuretics, which have some commonality with salt substitute in terms of their primary mechanism of action decreased blood pressure and slowed the pulse wave after long term use. ${ }^{27}$ Our study demonstrated that the 12 months salt substitute use could produce the similar effects and also suggested that arterial stiffness may be modified even in the patients with already severely impaired vasculature, by long-term 
intervention. Limited resources precluded the collection of $24 \mathrm{~h}$ urine specimens and this constrains our capability to study whether the effect was attributed to the reduction of sodium intake or the increase of potassium intake and by how much.

The response of the vasculature to different blood pressure-lowering interventions varies throughout the arterial tree and these differences are not always captured by measures of peripheral blood pressure. ${ }^{28,29}$ The effects of the salt substitute on central blood pressure demonstrated here provide further support for the expectation that salt substitution will provide vascular protection. ${ }^{19}$ As there are observational data linking central blood pressure more closely than peripheral blood pressure to target organ damage ${ }^{30}$ our findings on the effects of salt substitute on central blood pressure make a strong argument for beneficial effects beyond those that might be anticipated on the basis of the falls in blood pressure documented by brachial measurements. The same is true for the observed effects of the salt substitute on central pulse pressure, a measure that while strongly associated with the risk of death ${ }^{31}$ it is also associated closely with vascular disease outcomes. ${ }^{32}$

The salt substitute used in the study (65\% sodium chloride, $25 \%$ potassium chloride and $10 \%$ magnesium sulfate) would theoretically replace $35 \%$ of total salt intake in the intervention group. In the study reported here, the participants were all from Beijing, where the sodium intake was on average $275 \mathrm{mmolday}^{-1}$ according to our previous study. ${ }^{33}$ This means there should be $96 \mathrm{mmolday}^{-1}$ of sodium being replaced on average in theory. To maximize the use of salt substitute, we required, as a criteria for entry into the study, the participants must eat at least two of the three meals per day at home. However, we could not know exactly how much of sodium was reduced, although all participants (100\%) reported that they used study salt substitute/salt for 'all' or 'nearly all' of their day to day food preparation with no difference between randomized groups $(P=0.88)$. We agree that this is a limitation of our study but given the few resources available to us it was simply not possible for us to collect data of either complete diet or 24-h urine for precise estimates of sodium and potassium intake.

In conclusion, this study has reinforced the overall findings of the China Salt Substitute Study regarding the likely benefits of salt substitute-based blood pressure lowering on major vascular events and suggests that the immediate effects of salt substitute on vascular disease could be reliably estimated using measured effects on peripheral blood pressure attributable primarily to volume depletion and the long-term beneficial effects may come also from the reduction in arterial stiffness. In the meantime, salt substitute is a practical low cost alternate or complement to drug therapy for blood pressure control that would be practical and affordable for many in rural China. Nonoptimal blood pressure levels are a leading cause of disease burden in rural China and the reductions achieved with the widespread use of salt substitute would be substantial. ${ }^{33-35}$ Beneficial effects of salt substitute on blood pressure ${ }^{36}$ in conjunction with clear evidence of mortality benefits in large drug-based trials of blood pressure lowering ${ }^{37}$ serve to highlight the huge potential for reducing the cardiovascular disease burden in the rural Chinese setting with this under-utilized intervention strategy.

\section{ACKNOWLEDGEMENTS}

We are grateful to Mr Jianhua Qi and Dr Rujun Pan at the Yinhai Community Health Service Center, Daxing, Beijing and Mr Bin Li and Dr Zhigang Yang at the Fengbo Community Health Service Center, Shunyi, Beijing for their great contribution in collection of data in the study. We also thank Dr Michael O'Rourke at The Victor Change Cardiac Research Institute in the University of
New South Wales, for their careful review of the paper and valuable comments that helped to refine the paper. Bruce Neal was supported by a Career Development Award from the National Heart Foundation of Australia. The study was jointly funded by The Capital Medical Science Development Fund (China), The George Institute for International Health (Australia), The Clinical Trials Research Unit (New Zealand), and the National Health and Medical Research Council of Australia (Grant ID: 358395).

\section{CONFLICT OF INTEREST}

The authors declare no conflict of interest.

1 Cameron JD, Gataka CD, Kingwell BA. Assessment of large artery function. Coron Artery Dis 2002; 13: 405-413.

2 Meissner I, Khandheria BK, Sheps SG, Schwartz GL, Wiebers DO, Whisnant JP, Covalt JL, Petterson TM, Christianson TJ, Agmon Y. Atherosclerosis of the aorta: risk factor, risk marker, or innocent bystander? A prospective population-based transesophageal echocardiography study. J Am Coll Cardiol 2004; 44: 1018-1024.

3 Domanski MJ, Davis BR, Pfeffer MA, Kastantin M, Mitchell GF. Isolated systolic hypertension: prognostic information provided by pulse pressure. Hypertension 1999; 34: 375-380.

4 Chae CU, Pheffer MA, Glynn RJ, Mitchell GF, Taylor JO, Hennekens CH. Increased pulse pressure and risk of heart failure in the elderly. JAMA 1999; 281: 634-639.

5 O'Rourke MF, Yaginuma T. Wave reflections and the arterial pulse. Arch Intern Med 1984; 144: 366-371.

6 Laurent S, Boutouyrie P, Asmar R, Gautier I, Laloux B, Guize L, Ducimetiere P, Benetos A. Aortic stiffness is an independent predictor of all-cause and cardiovascular mortality in hypertensive patients. Hypertension 2001; 37: 1236.

7 Weber T, Auer J, O'Rourke MF, Kvas E, Lassnig E, Berent R, Eber B. Aortic stiffness, wave reflections, and the risk of coronary artery disease. Circulation 2004; 109: 184-189.

8 Hashimoto J, Imai Y, O'Rourke MF. Indices of pulse wave analysis are better predictors of left ventricular mass reduction than cuff pressure. Am J Hypertens 2007; 20: 378-384.

9 London GM, Blacher J, Pannier B, Guérin AP, Marchais SJ, Safar ME. Arterial wave reflections and survival in end-stage renal failure. Hypertension 2001; 38: 434-438.

10 Safar ME, London GM. Therapeutic studies and arterial stiffness in hypertension: recommendations of the European Society of hypertension. The Clinical Committee of Arterial Structure and Function. Working Group on Vascular Structure and Function of the European Society of Hypertension. J Hypertens 2000; 18: 1527-1535.

$11 \mathrm{He}$ FJ, MacGregor GA. Effect of longer-term modest salt reduction on blood pressure. Cochrane Database Syst Rev 2004; 3: CD004937.

12 He FJ, MacGregor GA. Effect of modest salt reduction on blood pressure: a metaanalysis of randomized trials. J Hum Hypertens 2002; 16: 761-770.

13 Karppanen H, Tanskanen A, Tuomilehto J, Puska P, Vuori J, Jäntti V, Seppänen ML. Safety and effects of potassium- and magnesium-containing low sodium salt mixtures. J Cardiovasc Pharmacol 1984; 6 (Suppl 1): S236-S243.

14 Geleijnse JM, Witteman JCM, Back AAA, Den Breeijen JH, Grobbee DE. Reduction in blood pressure with a low sodium, high potassium, high magnesium salt in older subjects with mild to moderate hypertension. BMJ 1994; 309: 436-440.

15 Kawasaki T, Itoh K, Kawasaki M. Reduction in blood pressure with a sodium-reduced, potassium-and magnesium-enriched mineral salt in subjects with mild essential hypertension. Hypertens Res 1998; 21: 235-243.

16 Avolio AP, Clyde KM, Beard TC, Cooke HM, Ho KK, O'Rourke MF. Improved arterial distensibility in normotensive subjects on a low salt diet. Arteriosclerosis 1986; 6: $166-169$.

17 Polonia J, Maldonado J, Ramos R, Bertoquini S, Duro M, Almeida C, Ferreira J, Barbosa L, Silva JA, Martins L. Estimation of salt intake by urinary sodium excretion in a Portuguese adult population and its relationship to arterial stiffness. Rev Port Cardiol 2006; 25: 801-817.

18 Dart AM, Qi XL. Determinants of arterial stiffness in Chinese migrants to Australia. Atherosclerosis 1995; 117: 263-272.

19 The China Salt Substitution Study Collaborative Group. Salt substitution: a low-cost strategy for blood pressure control among rural Chinese. A randomised controlled trial. J Hypertens 2007; 25: 2011-2018.

20 Pauca AL, O'Rourke MF, Kon ND. Prospective evaluation of a method for estimating ascending aortic pressure from the radial artery pressure waveform. Hypertension 2001; 38: 932-937.

21 Chen $\mathrm{CH}$, Nevo E, Fetics B, Pak PH, Yin FC, Maughan WL, Kass DA. Estimation of central aortic pressure waveform by mathematical transformation of radial tonometry pressure. Validation of generalised transfer function. Circulation 1997; 95: 1827-1836.

22 Gate PE, Tanaka H, Hiatt WR, Seals DR. Dietary sodium restriction rapidly improves large elastic artery compliance in older adults with systolic hypertension. Hypertension 2004; 44: 35-41.

23 Stewart AD, Jiang B, Millasseau SC, Ritter JM, Chowienczyk PJ. Acute reduction of blood pressure by nitroglycerin does not normalize large artery stiffness in essential hypertension. Hypertension 2006; 48: 404-410. 
24 Safar ME, Asmar R, Benetos A, Levy BI, London GM. Sodium, large arteries, and diuretic compounds in hypertension. Am J Med Sci 1994; 307 (Suppl 1): S3-S8.

25 Pannier B, Guérin A, London G, Asmar R, Safar M, REASON Study. Combination of lowdose perindopril/indapamide versus atenolol in the hypertensive patient. Effects on systolic pressure and arterial hemodynamics. REASON Study. Arch Mal Coeur Vaiss 2002; 95: 11-16.

26 Bénétos A, Laflèche A, Asmar R, Gautier S, Safar A, Safar ME. Arterial stiffness, hydrochlorothiazide and convertingenzyme inhibition in essential hypertension. J Hum Hypertens 1996; 10: 77-82.

27 Nichols WW, O'Rourke M. McDonald's Blood Flow in Arteries. Theoretical, Experimental and Clinical Principles, 4th edn. Arnold E: London, 1998, pp 54-113, 201222, 284-292, 347-401.

28 Hirata K, Kawakami M, O'Rourke MF. Pulse wave analysis and pulse wave velocity-a review of blood pressure interpretation 100 years after Korotkov. Circ J 2006; 70: 1231-1239.

29 Townsed RR. Analyzing the radial pulse waveform: narrowing the gap between blood pressure and outcomes. Curr Opin Nephrol Hypertens 2007; 16: 261-266.

30 Protogerou AD, Papaioannou TG, Blacher J, Papamichael CM, Lekakis JP, Safar ME. Central blood pressure: do we need them in the management of cardiovascular disease? Is a feasible therapeutic target? J Hypertens 2007; 25: 265-272.

31 Safar ME, Blacher J, Pannier B, Guerin AP, Marchais SJ, Guyonvarc'h PM, London GM. Central pulse pressure and mortality in end-stage renal disease. Hypertension 2002; 39: 735-738.
32 Roman MJ, Devereus RB, Kizer JR, Lee ET, Galloway JM, Ali T, Umans JG, Howard BV. Central pressure more strongly relates to vascular disease and outcome than does brachial pressure: the Strong Heart Study. Hypertens 2007; 50: 197-203.

33 Zhao L, Stamler J, Yan LL, Zhou B, Wu Y, Liu K, Daviglus ML, Dennis BH, Elliott P, Ueshima H, Yang J, Zhu L, Guo D. INTERMAP Research Group. Blood pressure differences between northern and southern Chinese: role of dietary factors: the International Study on Macronutrients and Blood Pressure. Hypertension 2004; 43 : 1332-1337.

34 China Ministry of Health, China Ministry of Science and Technology, and China State Bureau of Statisitics. The Nutrition and Health Status of the Chinese People. in Material for the Press Conference of the State Council Information Office, October 2004. Beijing, China.

35 Asaria P, Chisholm D, Mathers C, Ezzati M, Beaglehole R. Chronic disease prevention: health effects and financial costs of strategies to reduce salt intake and control tobacco use. Lancet 2007; 370: 2004.

$36 \mathrm{He}$ FJ, MacGregor GA. Salt, blood pressure and cardiovascular disease. Curr Opin Cardiol 2007; 22: 298-305.

37 Turnbull F. Blood Pressure Lowering Treatment Trialists' Collaboration. Effects of different blood-pressure-lowering regimens on major cardiovascular events: results of prospectively-designed overviews of randomised trials. Lancet 2003; 362: 1527-1535. 